\title{
THE EVOLUTION OF LINEARIZED PERTURBATIONS IN A MAGNETOHYDRODYNAMIC BOUNDARY LAYER
}

\author{
A.R. VIJAYALAKSHMI* \\ Department of Mathematics \\ Maharani's Science College for Women \\ Bangalore - 560 001, INDIA \\ E-mail: drarv@rediffmail.com \\ P.M. BALAGONDAR \\ Department of Mathematics, Central College \\ Bangalore University \\ Bangalore - 560 001, INDIA \\ E-mail:drp_mb@yahoo.com
}

\begin{abstract}
The evolution of linearized perturbations in a magnetohydrodynamic shear flow is studied using the initial value problem approach. Here the resulting equation in time posed by using the Fourier transform is solved for the Fourier amplitudes for modeled boundary layer for different initial disturbances. The shear flow prototype here is a piecewise linear approximation of a magnetohydrodynamic boundary layer. The initial disturbances that are considered are a point source of the field of transverse velocity and magnetic field. Solutions are obtained for small values of Alfve'n velocity. The velocity plots are drawn for different values of Alfve'n velocity.
\end{abstract}

Key words: magnetohydrodynamic boundary layer, initial value problem approach, Alfv'n velocity, Fourire transform technique.

\section{Introduction}

The stability of an electrically conducting shear flow is of interest to both geophysicists and astrophysicists.

The dynamics and stability of large-scale shear flows in a parallel magnetic field are of interest in astrophysical fluid dynamics. Anamalous transport rates are generally ascribed to turbulence, although in most of the presence of turbulence has not been established.

Stuart (1954) investigated the stability of a plane Poiseuille flow with the assumption that the mean magnetic field is everywhere constant. In this case the stability equation is similar to that of Orr-Sommerfeld equation with only one new term. Hains (1965) studied the influence of a coplanar magnetic field on the stability of a conducting fluid flowing between parallel planes. After deriving the general stability equation for small values of the magnetic Reynolds number, numerical results were obtained for the case where the initial perturbations of the magnetic field vanish. Hunt (1965) proved that when a uniform magnetic field is parallel to the flow and sufficiently large, the wave number vector of the most unstable disturbance is not, in general, parallel to the flow i.e., it is a three - dimensional disturbance. Lerner and Knobloch (1984) using the method of separation of variables studied the stability of a dissipative magnetohydrodynamic shear flow in a parallel magnetic field for the unbounded plane Couette flow. The finite conductivity and molecular viscosity were found to be stabilising. Venkatachalappa and Soward (1990) showed that the addition of small diffusivity, dissipation is strongly stabilising and there is an eventual collapse of all the modes. Kumari and

\footnotetext{
* To whom correspondence should be addressed
} 
Nath (1999) studied numerically the MHD Boundary layer flow of a non- Newtonian fluid over a continuously moving surface with a parallel free stream. Ruderman and Brevdo (2006) studied the stability of an MHD shear flow with piecewise linear velocity profile. They combined the analytical and numerical approaches to obtain the neutral stability curves in the case of both magnetic free flow and magnetic flow. Newton and Kim (2007) investigated numerically the dual role of shear flow in 2D MHD turbulence and the results indicated that the absence of resonance is responsible for the most catastropic reductions in transport. Douglas et al. (2008) studied the effects of flow shear and Alfven waves on two - dimensional magnetohydrodynamic turbulence and numerically found the underlying physical mechanisms for the reduction of turbulent transport and turbulence level by shear flow and magnetic field. Ruderman and Belov (2010) investigated the stability of MHD shear flows with application to space physics by considering heliopause stability and the stability of the Earth's magnetopause. Núñez, Manuel (2012) studied MHD shear flows with non - constant transverse magnetic field. They investigated the evolution of the flow and the magnetic field both near the plate and far from it by analyzing the possibility of reverse flow and the instability of the solutions. Mishnov et al. (2013) illustrated by numerical calculations and by exact solutions the time dependence of the magnetic field in a shear flow. Uddin (2013) studied numerically the MHD forced convective laminar Boundary layer flow from a convectively heated moving vertical plate with radiation and transpiration effect.

In the present paper, we have extended the work of Criminale and Drazin (1990) to magnetohydrodynamic shear flow. We have investigated the magnetohydrodynamic boundary layer, with unit pulse of velocity and magnetic field as initial conditions. The essence of the approach is as given below: For a multilayered basic flow with piecewise linear velocity profile, the complete general solution to the linearized equations of motion is obtained as a function of all space variables and time. The disturbances are resolved into rotational and irrotational components. The rotational solution is the solution for the hypothetical initial-value problem for which the mean flow is unbounded but coincides with the actual flow in the layer. The irrotational solution in each layer is specified uniquely by satisfying the interfacial conditions and boundary conditions at a wall or at infinity.

\section{Mathematical formulation}

We consider an electrically conducting fluid of density $\rho$, moving with velocity $\boldsymbol{q}$ in the presence of a magnetic field $\boldsymbol{H}$. Gravity is ignored. The governing equations of motion of an inviscid, incompressible, magnetohydrodynamic and Boussinesq fluid are

$$
\begin{aligned}
& \nabla \cdot \boldsymbol{q}=0, \\
& \nabla \cdot \boldsymbol{H}=0, \\
& \rho\left(\frac{\partial \boldsymbol{q}}{\partial t}+(\boldsymbol{q} \cdot \nabla) \boldsymbol{q}\right)=-\nabla P+\mu_{m}(\boldsymbol{H} \cdot \nabla) \boldsymbol{H}, \\
& \frac{\partial \boldsymbol{H}}{\partial t}+(\boldsymbol{q} \cdot \nabla) \boldsymbol{H}=(\boldsymbol{H} \cdot \nabla) \boldsymbol{q}
\end{aligned}
$$

where $P=p+\frac{\mu_{m} H^{2}}{2}$, is the total pressure, $\mu_{m}$ is the magnetic permeability.

The basic state of the system is $\boldsymbol{q}_{0}=(U(y)=\sigma y, 0,0), \boldsymbol{H}_{0}=\left(H_{0}, 0,0\right), P=P_{0}(y)$, where $\sigma$ is the shear of the mean flow. The quantities $\sigma$ and $H_{0}$ are assumed to be constants. 
In the study of linear stability, we superimpose a small wave like perturbation upon the mean flow i.e., $\boldsymbol{q}=\boldsymbol{q}_{0}+\boldsymbol{q}^{\prime}, \boldsymbol{H}=\boldsymbol{H}_{0}+\boldsymbol{H}^{\prime}, P=P_{0}+P^{\prime}$, where $\boldsymbol{q}^{\prime}, \boldsymbol{H}^{\prime}, P^{\prime}$ are the perturbed quantities of velocity, magnetic field and pressure respectively.

We linearize the above set of equations about the basic state then by employing (i) moving coordinates transformation, the frame of reference by defining $T=t, \xi=x-\sigma y t, \eta=y, \zeta=z$ (ii) three dimensional Fourier transformation given by $\hat{u}(\alpha ; \beta ; \gamma ; T)=\int_{-\infty}^{\infty} \int_{-\infty}^{\infty} \int_{-\infty}^{\infty} u(\xi ; \eta ; \zeta ; T) e^{i(\alpha \xi+\beta \eta+\gamma \zeta)} d \xi d \eta d \zeta$ with similar expressions for $\hat{v}, \hat{w}, \hat{H}_{x}, \hat{H}_{y}, \hat{H}_{z}$ and $\hat{P}$ (iii) Squire transformation defined by $\bar{\alpha}=\left(\alpha^{2}+\gamma^{2}\right)^{1 / 2}$ and $\varphi=\arctan (\gamma / \alpha)$, the velocity and magnetic field components in the $\bar{\alpha}$ and $\varphi$ directions are given by $\bar{u}=\frac{\alpha \hat{u}+\gamma \hat{w}}{\bar{\alpha}}, \bar{w}=\frac{-\gamma \hat{u}+\alpha \hat{w}}{\bar{\alpha}}, \bar{H}_{x}=\frac{\alpha \hat{H}_{x}+\gamma \hat{H}_{z}}{\bar{\alpha}}, \bar{H}_{z}=\frac{-\gamma \hat{H}_{x}+\alpha \hat{H}_{z}}{\bar{\alpha}}$. The resulting equations are

$$
\begin{aligned}
& \frac{d}{d T}\left(K^{2} \hat{v}\right)+i \alpha V_{A}^{2} K^{2} \hat{H}_{y}=0, \\
& \frac{d \hat{H}_{y}}{d T}=-i \alpha \hat{v}
\end{aligned}
$$

where $V_{A}^{2}=\frac{\mu_{m} H_{0}^{2}}{\rho_{0}}, V_{A}$ is the Alfve'n velocity, $\rho_{0}$ is the equilibrium density.

$$
K^{2}=\bar{\alpha}^{2}+(\beta-\sigma \alpha T)^{2} \quad \text { and } \quad \bar{\alpha}^{2}=\left(\alpha^{2}+\gamma^{2}\right) .
$$

The pressure amplitude $\hat{P}$ is obtained by taking the divergence of the momentum equations. It is found that $\hat{P}=\frac{-i 2 \sigma \alpha \hat{v}}{K^{2}}$ when $K^{2} \neq 0$.

Equation (2.5) admits two sets of solutions for $\hat{v}$, first, for $K^{2} \neq 0$, when the disturbance is rotational and second for $K^{2}=0$, when the disturbance is irrotational, since the vanishing of the product $k^{2} \hat{v}$ is equivalent to the Laplace equation $\nabla^{2} \hat{v}=0$ in real space. But for $\hat{H}_{y}$ a solution exists for only $K^{2} \neq 0$, since for $K^{2}=0, K^{2} \hat{H}_{y}=0$ corresponds to $\nabla^{2} \hat{H}_{y}=0$ which is equivalent to $\nabla \times \boldsymbol{H}=0$ and $\nabla \cdot \boldsymbol{H}=0$, which implies that $\boldsymbol{H}$ is a force free magnetic field, i.e., there is no magnetic field. Hence $\nabla^{2} \hat{H}_{y}=0$ which corresponds to the irratational solution, is not taken into account.

Now considering the case $K^{2} \neq 0$, we assume a perturbation solution for $\hat{v}_{R}$, the rotational component of $\hat{v}$ for small values of $V_{A}^{2}$ (Alfve'n velocity) in the form

$$
\begin{aligned}
& \hat{v}_{R}(\alpha, \beta, \gamma, T)=\hat{v}_{0}(\alpha, \beta, \gamma, T)+V_{A}^{2} \hat{v}_{1}(\alpha, \beta, \gamma, T)+\left(V_{A}^{2}\right)^{2} \hat{v}_{2}(\alpha, \beta, \gamma, T)+\ldots \\
& \hat{H}_{y}(\alpha, \beta, \gamma, T)=\hat{H}_{y 0}(\alpha, \beta, \gamma, T)+V_{A}^{2} \hat{H}_{y 1}(\alpha, \beta, \gamma, T)+\left(V_{A}^{2}\right)^{2} \hat{H}_{y 2}(\alpha, \beta, \gamma, T)+\ldots
\end{aligned}
$$


We find that

$$
\begin{aligned}
& \hat{v}_{0}=\frac{\hat{\Omega}_{0}(\alpha, \beta, \gamma)}{\bar{\alpha}^{2}+(\beta-\sigma \alpha T)^{2}}, \\
& \hat{v}_{1}=\left\{-\frac{\hat{\Omega}_{0} \bar{\alpha}^{3}}{\sigma}\left[\left(\frac{\beta-\sigma \alpha T}{\bar{\alpha}}\right) \tan ^{-1}\left(\frac{\beta-\sigma \alpha T}{\bar{\alpha}}\right)-\frac{1}{3} \log \left(\frac{\bar{\alpha}^{2}+(\beta-\sigma \alpha T)^{2}}{\bar{\alpha}^{2}}\right)+\right.\right. \\
& \left.+\frac{1}{3}\left(\frac{\beta-\sigma \alpha T}{\bar{\alpha}}\right)^{3} \tan ^{-1}\left(\frac{\beta-\sigma \alpha T}{\bar{\alpha}}\right)-\frac{1}{6}\left(\tan ^{-1}\left(\frac{\beta-\sigma \alpha T}{\bar{\alpha}}\right)\right)^{2}\right]+ \\
& \left.+\frac{i \bar{\alpha}^{3} \hat{\Omega}_{1}}{\sigma}\left(\left(\frac{\beta-\sigma \alpha T}{\bar{\alpha}}\right)+\frac{1}{3}\left(\frac{\beta-\sigma \alpha T}{\bar{\alpha}}\right)^{3}\right)\right\} \frac{1}{\bar{\alpha}^{2}+(\beta-\sigma \alpha T)^{2}}, \\
& \hat{v}_{2}=\left\{\left(\frac{\beta-\sigma \alpha T}{\bar{\alpha}}\right)^{3} \tan ^{-1}\left(\frac{\beta-\sigma \alpha T}{\bar{\alpha}}\right)\left(\frac{7 \bar{\alpha}^{5} \hat{\Omega}_{0}}{18 \sigma^{3} \alpha}\right)+\left(-\log \left(\frac{\bar{\alpha}^{2}+(\beta-\sigma \alpha T)^{2}}{\bar{\alpha}^{2}}\right)+\left(\frac{\beta-\sigma \alpha T}{\bar{\alpha}}\right)^{2}\right)\right. \\
& \left(\frac{5 \bar{\alpha}^{5} \hat{\Omega}_{0}}{36 \sigma^{3} \alpha}\right)+\left(\frac{\beta-\sigma \alpha T}{\bar{\alpha}}\right)^{5} \log \left(\frac{\bar{\alpha}^{2}+(\beta-\sigma \alpha T)^{2}}{\bar{\alpha}^{2}}\right)\left(\frac{\bar{\alpha}^{5} \hat{\Omega}_{0}}{10 \alpha^{3} \alpha}\right)+\left(\frac{\beta-\sigma \alpha T}{\bar{\alpha}}\right)^{4}\left(\frac{\bar{\alpha}^{5} \hat{\Omega}_{0}}{6 \sigma^{3} \alpha}\right)+ \\
& +\left(\frac{\beta-\sigma \alpha T}{\bar{\alpha}}\right)^{3}\left(-\frac{2 \bar{\alpha}^{5} \hat{\Omega}_{0}}{3 \sigma^{3} \alpha}+\frac{5 i \alpha \bar{\alpha}^{5} \hat{\Omega}_{1}}{27 \sigma^{2}}\right)+\left(\frac{\beta-\sigma \alpha T}{\bar{\alpha}}\right)\left(-\frac{2 \bar{\alpha}^{5} \hat{\Omega}_{0}}{\sigma^{3} \alpha}-\frac{8 i \alpha \bar{\alpha}^{5} \hat{\Omega}_{1}}{9 \sigma^{2}}\right)+ \\
& +\tan ^{-1}\left(\frac{\beta-\sigma \alpha T}{\bar{\alpha}}\right)\left(-\frac{2 \bar{\alpha}^{5} \hat{\Omega}_{0}}{\sigma^{3} \alpha}+\frac{8 i \alpha \bar{\alpha}^{5} \hat{\Omega}_{1}}{9 \sigma^{2}}\right)+\left(\frac{\beta-\sigma \alpha T}{\bar{\alpha}}\right) \tan ^{-1}\left(\frac{\beta-\sigma \alpha T}{\bar{\alpha}}\right)\left(\frac{2 \bar{\alpha}^{5} \hat{\Omega}_{0}}{3 \alpha^{3} \alpha}\right)+ \\
& +\left(\frac{\beta-\sigma \alpha T}{\bar{\alpha}}\right)^{5}\left(\frac{i \alpha \bar{\alpha}^{5} \hat{\Omega}_{1}}{\sigma^{2}}\right)+\left(\left(\frac{\beta-\sigma \alpha T}{\bar{\alpha}}\right)+\left(\frac{\beta-\sigma \alpha T}{\bar{\alpha}}\right)^{3}\right) \log \left(\frac{\bar{\alpha}^{2}+(\beta-\sigma \alpha T)^{2}}{\bar{\alpha}^{2}}\right) \\
& \left.\left(\frac{2 i \alpha \bar{\alpha}^{5} \hat{\Omega}_{l}}{3 \sigma^{2}}\right)\right\} \frac{1}{\bar{\alpha}^{2}+(\beta-\sigma \alpha T)^{2}} \text {. }
\end{aligned}
$$

The solution for $K^{2}=0$ is found by considering the perturbation equations where a two dimensional Fourier transform is used instead of the full three - dimensional decomposition. Using the moving co-ordinate transformation, $K^{2} \hat{v}=0$ corresponds to

$$
\frac{\partial^{2} \breve{v}_{I}}{\partial \eta^{2}}+2 i \sigma \alpha T \frac{\partial \breve{v}_{I}}{\partial \eta}-\left(\bar{\alpha}^{2}+\sigma^{2} \alpha^{2} T^{2}\right) \breve{v}_{I}=0
$$

where

$$
\breve{v}_{I}=\breve{v}_{I}(\alpha, \eta, \gamma ; T)=\int_{-\infty}^{\infty} \int_{-\infty}^{\infty} v_{I}(\xi, \eta, \varsigma, T) e^{i(\alpha \xi+\gamma \zeta)} d \xi d \zeta
$$

is the irrotational part of $v$. The solution of Eq.(2.12) is found to be 


$$
\breve{v}_{I}=A(T) e^{-\bar{\alpha} \eta-i \sigma \alpha T \eta}+B(T) e^{\bar{\alpha} \eta-i \sigma \alpha T \eta}
$$

where $A(T)$ and $B(T)$ are constants of integration .

In order to combine $\hat{v}_{R}$ and $\breve{v}_{I}$ to obtain the complete solution and satisfy the matching condition $\hat{v}_{R}$ must be inverted once to obtain $\breve{v}_{R}(\alpha, \eta, \gamma ; T)$, i.e.,

$$
\breve{v}_{R}(\alpha, \eta, \gamma ; T)=\frac{1}{2 \pi} \int_{-\infty}^{\infty} \hat{v}_{R}(\alpha, \beta, \gamma ; T) e^{-i \beta \eta} d \beta .
$$

With the initial velocity and initial magnetic field given by

$$
\begin{aligned}
& v(x, y, z, 0)=V_{0} \delta\left(x-x_{0}\right) \delta\left(y-y_{0}\right) \delta\left(z-z_{0}\right), \\
& H_{y}(x, y, z, 0)=H_{0} \delta\left(x-x_{0}\right) \delta\left(y-y_{0}\right) \delta\left(z-z_{0}\right) .
\end{aligned}
$$

In terms of moving co-ordinates and the three-dimensional Fourier transform

$$
\begin{aligned}
& \breve{v}_{0}(\alpha, \beta, \gamma)=\Omega_{0}(\alpha, \beta, \gamma)=V_{0} e^{i\left(\alpha x_{0}+\beta y_{0}+\gamma z_{0}\right)}, \\
& \breve{H}_{y 0}(\alpha, \beta, \gamma)=\Omega_{l}(\alpha, \beta, \gamma)=\tilde{H}_{0} e^{i\left(\alpha x_{0}+\beta y_{0}+\gamma z_{0}\right)},
\end{aligned}
$$

$\breve{v}_{R}$ is found to be

$$
\begin{aligned}
& \breve{v}_{R}=e^{i\left(\alpha x_{0}+\gamma z_{0}-\sigma \alpha T \bar{\eta}\right)}\left\{V_{0}\left(1-\frac{V_{A}^{2} \bar{\alpha}^{3}}{6 \sigma \alpha}-\frac{V_{A}^{4} \bar{\alpha}^{5}}{36 \sigma^{3} \alpha}\right) e^{-\bar{\alpha}|\bar{\eta}|}+V_{0}\left(\frac{2 V_{A}^{2} \bar{\alpha}^{3}}{3 \sigma}+\frac{5 V_{A}^{4} \bar{\alpha}^{5}}{18 \sigma^{3} \alpha}\right)\right. \\
& \int_{-\infty}^{\infty} \frac{\eta^{\prime} e^{-\bar{\alpha}\left|\bar{\eta}-\eta^{\prime}\right|-\bar{\alpha}|\bar{\eta}|}}{\left(\bar{\eta}-\eta^{\prime}\right)} d \eta^{\prime}+V_{0}\left(\frac{V_{A}^{2} \bar{\alpha}^{3}}{3 \sigma}-\frac{5 V_{A}^{4} \bar{\alpha}^{5}}{36 \sigma^{3} \alpha}\right) \int_{-\infty}^{\infty} \frac{e^{-\bar{\alpha}\left|\bar{\eta}-\eta^{\prime}\right|-\bar{\alpha}|\bar{\eta}|}}{\eta^{\prime}} d \eta^{\prime}+ \\
& +\left(\frac{2 i \bar{\alpha}^{3} \tilde{H}_{0}}{3 \sigma}-\frac{4 \bar{\alpha}^{5} V_{0}}{3 \sigma^{3} \alpha}+\frac{46 i \alpha \bar{\alpha}^{3} \tilde{H}_{0}}{27 \sigma^{2}}\right)\left(\frac{-i \bar{\eta}}{2} e^{-\bar{\alpha}|\bar{\eta}|}\right)+\left(-\frac{2 \bar{\alpha}^{5} V_{0}}{\sigma^{3} \alpha}+\frac{8 i \alpha \bar{\alpha}^{5} \tilde{H}_{0}}{9 \sigma^{2}}\right) \\
& \left.\left(\int_{-\infty}^{\infty}-i \frac{e^{-\bar{\alpha}\left(\bar{\eta}-\eta^{\prime}\right)-\bar{\alpha} \eta^{\prime}}}{\eta^{\prime}} d \eta^{\prime}\right)-\left(\frac{\bar{\alpha}^{5} V_{0}}{10 \sigma^{3} \alpha}-\frac{4 i \alpha \bar{\alpha}^{5} \tilde{H}_{0}}{9 \sigma^{2}}\right)\left(\int_{-\infty}^{\infty} i \frac{e^{-\bar{\alpha}\left|\bar{\eta}-\eta^{\prime}\right|-\bar{\alpha}\left|\eta^{\prime}\right|}}{2\left(\bar{\eta}-\eta^{\prime}\right)} d \eta^{\prime}\right)\right\} .
\end{aligned}
$$

Now the complete solution will be

$$
\breve{v}=\breve{v}_{R}+\breve{v}_{I}
$$

$\breve{v}_{R}$ and $\breve{v}_{I}$ are given by Eqs (2.19) and (2.14). 


\section{Magnetohydrodynamic boundary layer flow}

This problem consists of both the plane Couette flow and the layered infinite flow, i.e., wall layer (or shear layer) and the free stream. The model of the magnetohydrodynamic boundary layer is as shown in Fig.1.

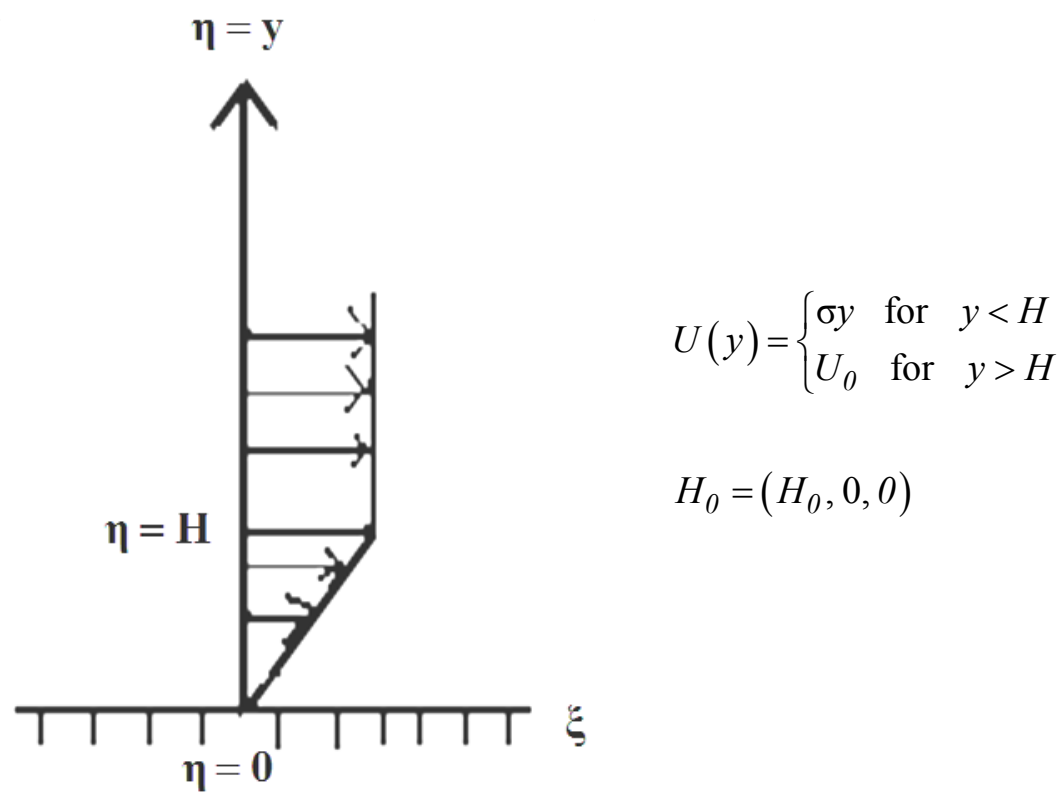

Fig.1. Sketch of magnetohydrodynamic boundary layer flow on a wall.

In the free stream, the moving co-ordinate system for a constant mean flow $U_{0}$ is given by

$$
T=t, \quad \xi=x-U_{0} t, \quad \eta=y, \quad \zeta=z .
$$

The Fourier transforms and inversions follow as it has been done before. Thus

$$
\begin{aligned}
& \breve{v}_{I_{S}}=A_{S}(T) e^{-\bar{\alpha} \eta} \\
& \breve{v}_{R_{S}}=e^{i\left(\alpha x_{0}+\gamma z_{0}\right)}\left[\left(V_{0}-\frac{i \alpha V_{A}^{2} T^{2}}{2}-\frac{i \alpha^{3} V_{A}^{4} T^{2} V_{0}}{24}\right)\right] e^{-\bar{\alpha} \bar{\eta}} .
\end{aligned}
$$

The subscript $s$ denotes the quantities in the free stream. In the wall layer, $\breve{v}_{R}$ and $\breve{v}_{I}$ are given by Eqs (2.20) and (2.14).

We have $\breve{v}=0$ at the solid boundary $\eta=0$. Thus,

$$
A+B=-A_{11} e^{i\left(\alpha x_{0}+\gamma z_{0}+\sigma \alpha T y_{0}\right)} .
$$

We make use of the kinematic condition and pressure matching conditions.

Velocity $\breve{v}$ must be continuous at the interface $\eta=H$, i.e., $\left[\breve{v}_{s}\right]_{\eta=H}=[\breve{v}]_{\eta=H}$ gives 


$$
\begin{aligned}
& A_{S}(T) e^{-\bar{\alpha} H}+e^{i\left(\alpha x_{0}+\gamma z_{0}\right)-\bar{\alpha}\left(H-y_{0}\right)} V_{0}\left(1-\frac{i \alpha V_{A}^{2} T^{2}}{2}-\frac{i \alpha^{3} V_{A}^{4} T^{3}}{12}\right)= \\
& =\left(A e^{\bar{\alpha} H}+B e^{-\bar{\alpha} H}\right) e^{-i \sigma \alpha T\left(H-y_{0}\right)}+A_{9} e^{i\left(\alpha x_{0}+\gamma z_{0}-\sigma \alpha T\left(H-y_{0}\right)\right)} .
\end{aligned}
$$

Pressure $\hat{P}$ must be continuous at the interface $\eta=H$, i.e., $\left[\breve{p}_{\text {free stream }}\right]_{\eta=H}\left[\breve{p}_{\text {wall layer }}\right]_{\eta=H}$ gives

$$
\begin{aligned}
& -\bar{\alpha} A_{S}(T) e^{-\bar{\alpha} \eta}+-\bar{\alpha} e^{i\left(\alpha x_{0}+\gamma z_{0}\right)-\bar{\alpha}\left(H-y_{0}\right)}\left(-i \alpha V_{A}^{2} V_{0}-\frac{i \alpha^{3} V_{A}^{4} T^{3}}{12} \tilde{H}_{0}\right)= \\
& =\left[\left(-\dot{A} e^{-\bar{\alpha} H}+\dot{B} e^{+\bar{\alpha} H}\right) \bar{\alpha}+i \sigma \alpha(1+\bar{\alpha} H) A e^{-\bar{\alpha} H}+i \sigma \alpha(1-\bar{\alpha} H) B e^{\bar{\alpha} H}\right] e^{-i \sigma \alpha T H}+F_{5} e^{i \sigma \alpha T H} .
\end{aligned}
$$

From Eqs (3.5) and (3.6), we obtain

$$
\begin{aligned}
& B=\frac{1}{2 \bar{\alpha}}\left[\frac{\alpha A_{11} e^{-2 \bar{\alpha} H-i \sigma \alpha T_{y}}}{\sigma\left(\frac{1-2 \bar{\alpha} H+e^{-2 \bar{\alpha} H}}{2 \bar{\alpha}}-\alpha y_{0}\right)}+\frac{\left(F_{5}+i \sigma \alpha A_{9} e^{i \sigma \alpha T y_{0}}\right) e^{-\bar{\alpha} H-i \sigma \alpha T H}}{i \sigma\left(\frac{1-2 \bar{\alpha} H+e^{-2 \bar{\alpha} H}}{2 \bar{\alpha}}-\alpha\left(H-y_{0}\right)\right)}\right] \\
& e^{i \sigma\left(\frac{1-2 \bar{\alpha} H+e^{-2 \bar{\alpha} H}}{2 \bar{\alpha}}-\alpha y_{0}\right) T} e^{i\left(\alpha x_{0}+\gamma z_{0}\right)}+D_{3} e^{i \sigma\left(\frac{1-2 \bar{\alpha} H+e^{-2 \bar{\alpha} H}}{2 \bar{\alpha}}-\alpha y_{0}\right) T} .
\end{aligned}
$$

Using $B(0)=0, D_{3}$ is found to be

$$
D_{3}=-\frac{e^{i\left(\alpha x_{0}+\gamma_{0}\right)}}{2 \bar{\alpha}}\left[\frac{\alpha A_{1 l} e^{-2 \bar{\alpha} H}}{\sigma\left(\frac{1-2 \bar{\alpha} H+e^{-2 \bar{\alpha} H}}{2 \bar{\alpha}}-\alpha y_{0}\right)}+\frac{\left(F_{5}+i \sigma \alpha A_{9}\right) e^{-\alpha H}}{i \sigma\left(\frac{1-2 \bar{\alpha} H+e^{-2 \bar{\alpha} H}}{2 \bar{\alpha}}-\alpha\left(H-y_{0}\right)\right)}\right] .
$$

Equation (3.4) yields

$$
A=-A_{11} e^{i\left(\alpha x_{0}+\gamma z_{0}+\sigma \alpha T y_{0}\right)}-B
$$

The values of the coefficients are given in Appendix.

\section{Results and discussions}

In this problem, we have studied the evolution of linearized perturbations of a basic flow of an inviscid magnetohydrodynamic shear flow using piecewise linear velocity profiles. We have used unit pulse for velocity and magnetic field as initial distributions. The systematic development is in principle can be applicable to any basic flow with piecewise linear velocity profile, but we have concentrated on magnetohydrodynamic boundary layer flow. 
In these broken line (piecewise linear) profiles, we have resolved the perturbations into rotational and irrotational components. Plots are drawn to observe the variation of amplitude of rotational velocity $\left|\hat{v}_{R}\right|$ and magnetic field $\left|\hat{H}_{y}\right|$ with time. Figures 2a-2d are plots of $\left|\hat{v}_{R}\right| V_{S} T$ for different values of $V_{A}$ $\left(V_{A}=0,0.2,0.5\right)$ and $\varphi\left(\varphi=0^{\circ}, 45^{\circ}, 90^{\circ}, 180^{\circ}\right)$. As time increases there is a decay in $\left|\hat{v}_{R}\right|$.

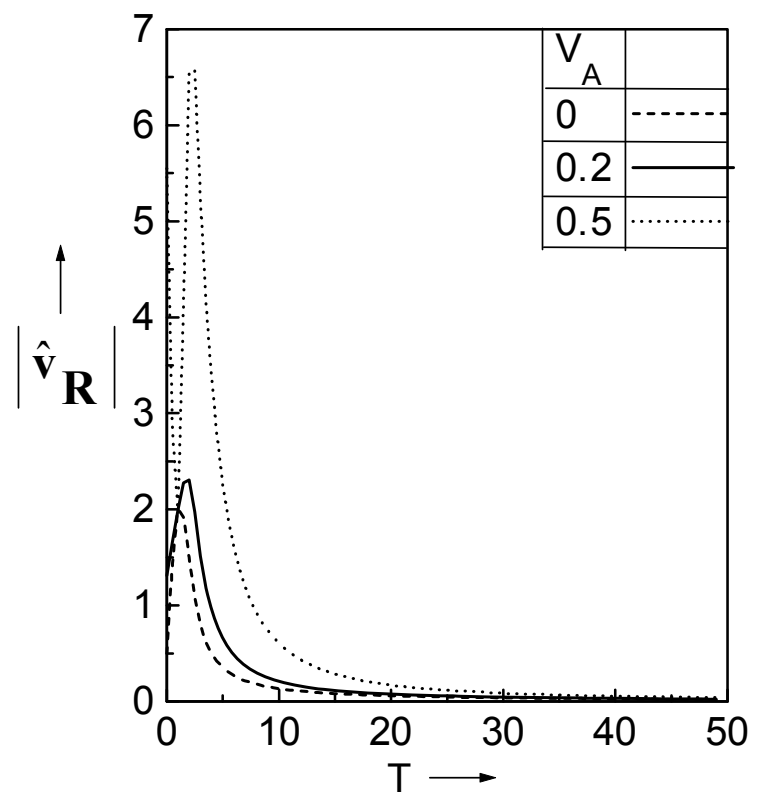

(a)

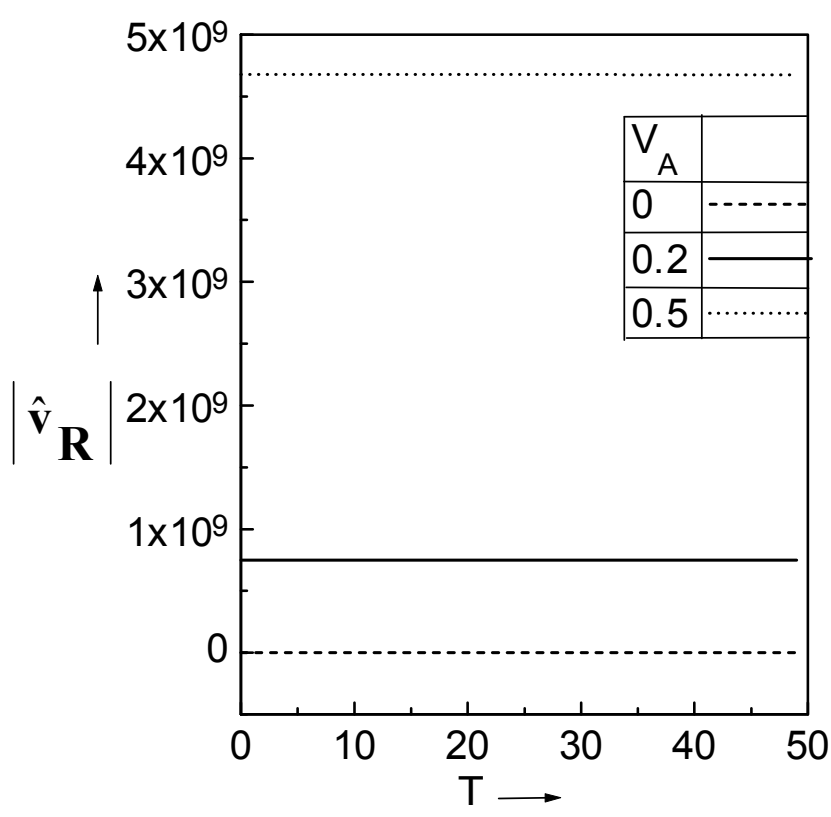

(c)

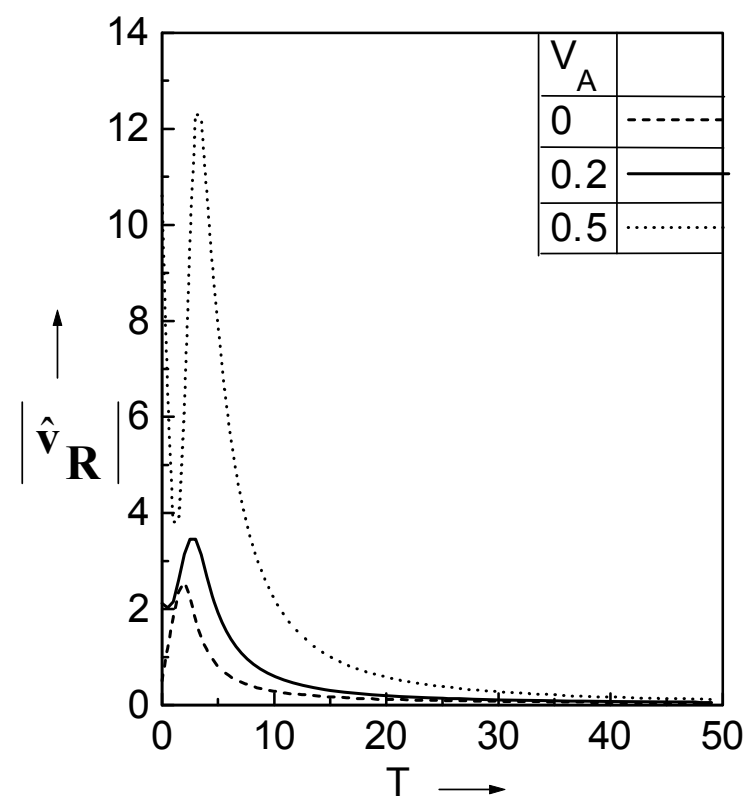

(b)

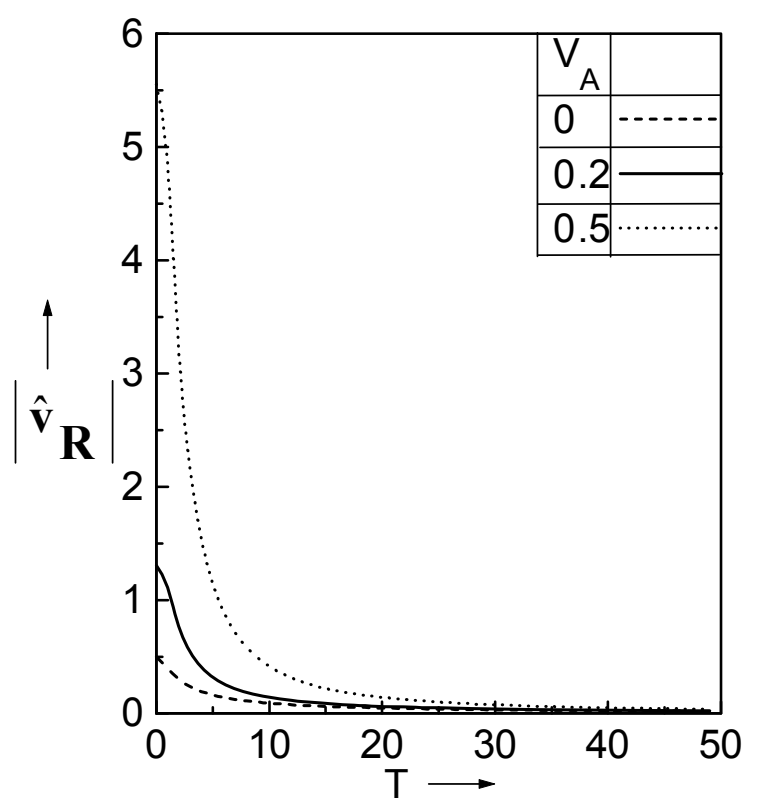

(d)

Fig.2. Curves of $\left|\hat{v}_{R}\right|$ versus $T$ for (a) $\varphi=0^{\circ}, \varphi=0^{\circ}$, (b) $\varphi=45^{\circ}$, (c) $\varphi=90^{\circ}$ and (d) $\varphi=180^{\circ}$ for different values of $V_{A}$. 


\section{Nomenclature}

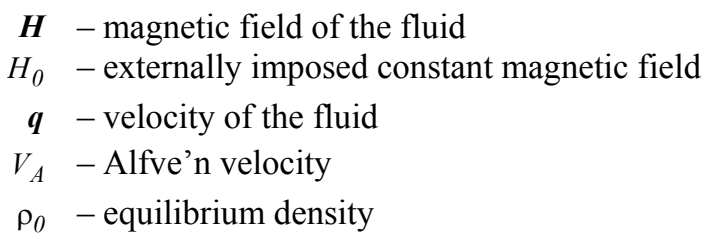

\section{References}

Criminale W.O. and Drazin P.G. (2000): The initial-value problem for a modeled boundary layer. - Phys. Fluids, vol.12(2), p.366.

Douglas J., 1 Eun-jin Kim and Thyagaraja A. (2008): Effects of flow shear and Alfvén waves on two-dimensional magnetohydrodynamic turbulence. - Physics of Plasmas 15, 23.

Hains F.D. (1965): Stability diagrams for magnetogasdynamic channel flow. - Phys. Fluids, vol.8(11), p.2014.

Hunt J.C.R. (1966): On the stability of parallel flows with parallel magnetic field. - Proc. R. Soc. Lond. A, vol.293, p.342.

Knobloch K. (1984): The stability of stratified shear flow. - Geophysics and Astrophysics, Fluid Dynamics, vol.29, p.105.

Kumari M. and Nath G. (1999): MHD Boundary layer flow of a non-Newtonian fluid over a continuously moving surface with a parallel free stream. - Acta Mechanica, 146, 139.

Lerner J. and Knobloch E. (1985): The stability of dissipative magnetohydro-dynamic shear flow in a parallel magnetic field. - Geophysics and Astrophysics, Fluid Dynamics, vol.33, pp.295-314.

Mishonov T.M., Maneva Y.G., Dimitrov Z.D. and Hristov T.S. (2013): On the theory of MHD waves in a shear flow of a magnetized turbulent plasma. - Astrophysics. 1,12.

Newton A.P and Kim E.J. (2009): Investigation into the dual role of shear flow in 2D MHD turbulence. - Phys. Rev. Lett. Apr. 24; 102(16):165002.

Núñez, Manuel (2012): MHD shear flows with non-constant transverse magnetic field. - Physics Letters A, 376, 19, 1624.

Ruderman M.S. and Brevdo L. (2006): Stability of an MHD shear flow with a piecewise linear velocity profile. Astron. and Astrophys., 448, 1177.

Ruderman M.S. and Belov N.A. (2010): Stability of MHD shear flows: Application to space physics. - Journal of Physics: Conference Series, vol.216, 1.

Stuart J.T. (1954): On the stability of viscous flow between parallel planes in the presence of co-planar magnetic field. - Proc. R. Soc. Lond., vol.A 221, p.189.

Uddin Md. Jashim, Waqar A. Khan mail and Ismail A.I. Md. (2013): MHD Forced Convective Laminar Boundary Layer Flow from a Convectively Heated Moving Vertical Plate with Radiation and Transpiration Effect. - PLOS ONE, 20.

Venkatachalappa M. and Soward A.M. (1990): The stability of stratified conducting shear flow in an aligned magnetic field. - Geophysics and Astrophysics, Fluid Dynamics, vol.54, p.109. 


\section{Appendix}

$$
\begin{aligned}
& A_{9}=V_{0}\left(1-\frac{V_{A}^{2} \bar{\alpha}^{3}}{6 \sigma \alpha}-\frac{V_{A}^{4} \bar{\alpha}^{5}}{36 \sigma^{3} \alpha}\right) e^{-\bar{\alpha}\left|H-y_{0}\right|_{+}} \\
& +V_{0}\left(\frac{2 V_{A}^{2} \bar{\alpha}^{3}}{3 \sigma}+\frac{5 V_{A}^{4} \bar{\alpha}^{5}}{18 \sigma^{3} \alpha}\right) \int_{-\infty}^{\infty} \frac{\eta^{\prime} e^{-\bar{\alpha}\left|H-y_{0}-\eta^{\prime}\right|-\bar{\alpha}\left|\eta^{\prime}\right|}}{\left(H-y_{0}-\eta^{\prime}\right)} d \eta^{\prime}+ \\
& +V_{0}\left(\frac{V_{A}^{2} \bar{\alpha}^{3}}{3 \sigma}-\frac{5 V_{A}^{4} \bar{\alpha}^{5}}{36 \sigma^{3} \alpha}\right) \int_{-\infty}^{\infty} \frac{e^{-\bar{\alpha}\left|H-y_{0}-\eta^{\prime}\right|-\bar{\alpha}\left|\eta^{\prime}\right|}}{\eta^{\prime}} d \eta^{\prime}+\left(\frac{2 i \bar{\alpha}^{3} \tilde{H}_{0}}{3 \sigma}-\frac{4 \bar{\alpha}^{5} V_{0}}{3 \sigma^{3} \alpha}+\frac{46 i \alpha \bar{\alpha}^{3} \tilde{H}_{0}}{27 \sigma^{2}}\right) \\
& \left(\frac{-i\left(H-y_{0}\right)}{2} e^{-\bar{\alpha}\left|H-y_{0}\right|}\right)-\left(-\frac{2 \bar{\alpha}^{5} V_{0}}{\sigma^{3} \alpha}+\frac{8 i \alpha \bar{\alpha}^{3} \tilde{H}_{0}}{9 \sigma^{2}}\right)\left(\int_{-\infty}^{\infty} i \frac{e^{-\bar{\alpha}\left(H-y_{0}-\eta^{\prime}\right)-\bar{\alpha} \eta^{\prime}}}{\eta^{\prime}} d \eta^{\prime}\right)+ \\
& \left.-\left(\frac{\bar{\alpha}^{5} V_{0}}{10 \sigma^{3} \alpha}-\frac{4 i \alpha \bar{\alpha}^{5} \tilde{H}_{0}}{9 \sigma^{2}}\right)\left(\int_{-\infty}^{\infty} i \frac{e^{-\bar{\alpha}\left|H-y_{0}-\eta^{\prime}\right|-\bar{\alpha}\left|\eta^{\prime}\right|}}{2\left(H-y_{0}-\eta^{\prime}\right)} d \eta^{\prime}\right)\right\}, \quad A_{11}=\left.A_{9}\right|_{H=0}, \\
& F_{5}=e^{i\left(\alpha x_{0}+\gamma z_{0}+\sigma \alpha T y_{0}\right)}\left\{V_{0}\left(1-\frac{V_{A}^{2} \bar{\alpha}^{3}}{6 \sigma \alpha}-\frac{V_{A}^{4} \bar{\alpha}^{5}}{36 \sigma^{3} \alpha}\right) e^{-\bar{\alpha}\left|H-y_{0}\right|}\left(1-\bar{\alpha}\left(H-y_{0}\right)\right)+\left(\frac{8 \alpha \bar{\alpha}^{5} \tilde{H}_{0}}{9 \sigma^{2}}+\right.\right. \\
& +V_{0}\left(\frac{V_{A}^{2} \bar{\alpha}^{3}}{3 \sigma}-\frac{5 V_{A}^{4} \bar{\alpha}^{5}}{18 \sigma^{3} \alpha}+\frac{2 i \bar{\alpha}^{5}}{\sigma^{3} \alpha}\right) \int_{-\infty}^{\infty} \frac{e^{-\bar{\alpha}\left|H-y_{0}-\eta^{\prime}\right|-\bar{\alpha} \mid} \eta^{\prime} \mid}{\eta^{\prime}}\left(\frac{1-\bar{\alpha}\left(H-y_{0}\right)}{\left(H-y_{0}-\eta^{\prime}\right)}\right) d \eta^{\prime}+\left(\frac{2 V_{A}^{2} \bar{\alpha}^{3} V_{0}}{3 \sigma}+\right.
\end{aligned}
$$

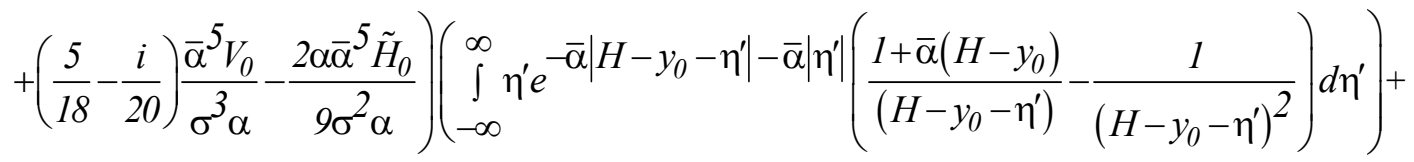

$$
\begin{aligned}
& \left.+\left(\frac{\bar{\alpha}^{3} \tilde{H}_{0}}{\sigma}+\frac{2 i \bar{\alpha}^{5} V_{0}}{3 \sigma^{3} \alpha}+\frac{23 \alpha \bar{\alpha}^{5} \tilde{H}_{0}}{9^{\sigma 2}}\right) e^{-\bar{\alpha}\left|H-y_{0}-\eta^{\prime}\right|}\left(\bar{\alpha}\left(H-y_{0}\right)\right)\right\}(-i \sigma \alpha)+\left\{\left(\frac{2 V_{A}^{2} \alpha \bar{\alpha}^{2} \tilde{H}_{0}}{3 \sigma}+\right.\right. \\
& +V_{0}\left(\frac{1}{\sigma}-\frac{2 V_{A}^{2} \bar{\alpha}^{2}}{3 \sigma^{2} \alpha}-\frac{i V_{A}^{4} \bar{\alpha}^{4}}{90 \sigma^{4} \alpha^{2}}\right)+e^{-\bar{\alpha}\left|H-y_{0}\right|}\left(\tilde{H}_{0}\left(-\frac{2 i \bar{\alpha}^{4}}{9 \sigma^{3}}+\frac{2 i \bar{\alpha}^{4}}{276 \sigma^{2}}+\frac{4 \bar{\alpha}^{4} V_{0}}{15 \sigma^{4} \alpha^{2}}\right) V_{A}^{4}\right)\left(-i \alpha V_{A}^{2}\right) \\
& \left(\frac{\bar{\alpha}}{\left(H-y_{0}\right)}+\frac{1}{\left(H-y_{0}\right)^{2}}\right)+\left(V_{A}^{4}\left(-\frac{4 i \bar{\alpha}^{4} V_{0}}{20 \sigma^{4} \alpha^{2}}+\frac{6 i \bar{\alpha}^{4} \tilde{H}_{0}}{9 \sigma^{2}}\right)-\frac{i \bar{\alpha}^{4} V_{0}}{3 \sigma^{4} \alpha^{2}}\right) \int_{-\infty}^{\infty} e^{-\bar{\alpha}\left|-H-y_{0}-\eta^{\prime}\right|-\bar{\alpha}|\bar{\eta}|} \\
& \left.\left(-i \alpha V_{A}^{2}\right)\left(\frac{\bar{\alpha}}{\left(H-y_{0}-\eta^{\prime}\right)}+\frac{1}{\left(H-y_{0}-\eta^{\prime}\right)^{2}}\right) d \eta^{\prime}\right\} \text {. }
\end{aligned}
$$

Received: September 23, 2013

Revised: March 2, 2014 